\title{
Impacto de la medicina nuclear en el diagnóstico y tratamiento del cáncer diferenciado de tiroides
}

\author{
Sevastián Medina-Ornelas, ${ }^{1}$ Francisco García-Pérez ${ }^{1}$ y Martín Granados-García ${ }^{2}$ \\ ${ }^{1}$ Departamento de Medicina Nuclear e Imagen Molecular; ${ }^{2}$ Departamento de Cirugía de Cabeza y Cuello. Secretaría de Salud, Instituto Nacional \\ de Cancerología, Ciudad de México, México
}

\begin{abstract}
Resumen
Los pacientes afectados por el cáncer diferenciado de tiroides habitualmente presentan un curso clínico favorable, ya que la piedra angular del tratamiento es la cirugía; a pesar de esto, algunos pueden desarrollar un ominoso desenlace, debido a las características clinico-patológicas de esta enfermedad. El tratamiento óptimo aún es controvertido, en especial respecto a la extensión de la cirugía, indicaciones de radioyodo y la supresión de la hormona estimulante de la tiroides. La correcta evaluación de los riesgos, antes y después de la cirugía, facilita un selectivo enfoque del tratamiento; destacando la relevancia de revisar el impacto de la medicina nuclear en la correcta evaluación, tratamiento y seguimiento de los pacientes que padecen esta neoplasia.
\end{abstract}

PALABRAS CLAVE: Cáncer tiroides. Radioyodo. Medicina nuclear.

\begin{abstract}
Patients affected by differentiated thyroid cancer usually have a favorable clinical course, since the cornerstone of treatment is surgery; despite this, some patients may develop an ominous outcome, due to the clinical-pathological features of this disease. Optimal treatment remains controversial, especially regarding the extent of surgery, indications for radioiodine and thyroid-stimulating hormone. The correct evaluation of risks before and after surgery facilitates a selective treatment approach; highlighting the importance of reviewing the impact of nuclear medicine on the correct evaluation, treatment and follow-up of patients suffering from this neoplasm.
\end{abstract}

KEY WORDS: Thyroid cancer. Radioiodine. Nuclear medicine.

\section{Introducción}

Según Globocan, en 2012 en México ocurrieron 3036 casos de cáncer de tiroides (725 en hombres y 2311 en mujeres), que constituyeron $2.1 \%$ del total de neoplasias malignas. ${ }^{1}$ El cáncer diferenciado de tiroides (CDT) representa al menos $85 \%$ de los casos e incluye los tipos papilar, folicular y de células de Hürtle. El cáncer papilar, el tipo más frecuente, se asocia con excelente supervivencia, pero algunos pacientes tienen un curso clínico menos favorable, con metástasis o recaídas que afectan los ganglios linfáticos cervicales y con menor frecuencia, pulmones y hueso. ${ }^{2,3}$
La medicina nuclear emplea las peculiaridades de la fisiopatología del CDT y desempeña un papel muy importante, tanto en el tratamiento adyuvante como en el seguimiento y manejo de las recaídas. El tratamiento debe ser el más eficaz, con las menores secuelas y menor costo, por ello se hace énfasis en la individualización acorde al tipo histológico, extensión de la enfermedad y riesgo de recaída.

\section{El yodo 131}

El yodo 131 (131), radioyodo o RAI) es producido en un reactor nuclear mediante irradiación neutrónica del dióxido de telurio durante la fisión del uranio. La vida
Correspondencia: Sevastián Medina-Ornelas E-mail: dr.sevastian@outlook.com
Fecha de recepción: 23-01-2017

Fecha de aceptación: 08-01-2018 DOI://dx.doi.org/10.24875/GMM.18003206
Gac Med Mex. 2018;154:509-519

Disponible en PubMed www.gacetamedicademexico.com 
media física es de 8.02 días. Los átomos del yodo 131 emiten partículas $\beta$ con varias energías, la máxima de $606 \mathrm{keV}$, fundamental para el efecto terapéutico del yodo 131; después de la emisión de la partícula $\beta$, emite rayos $\gamma$ con energía de 364 a 637 $\mathrm{keV}$, útil para obtener imágenes gammagráficas o mediante tomografía computarizada de emisión monofónica (SPECT). El yodo 131 se dispensa en forma de ioduro líquido 0 en cápsulas para su ingesta oral. ${ }^{4}$

\section{Radiobiología}

La mayor radiación emitida por el yodo 131 ocurre en la forma de partículas $\beta$ que interrumpen los enlaces químicos e infligen daño devastador al ADN, que produce disfunción celular y activación de la muerte celular programada. Esto sucede mediante dos vías: la primera o directa resulta de la irradiación de las partículas $\beta$ sobre la cadena simple del ADN, mientras que la segunda, o indirecta, ocurre mediante la interacción con moléculas de agua que forma radicales libres, mismos que dañan el ADN; esta última es la más importante debido al mayor daño infringido. ${ }^{5}$

Las partículas $\beta$ penetran el tejido un par de milímetros. La dosis absorbida y entregada por las partículas $\beta$ para una determinada dosis radiactiva aumenta con el radio de tejido hasta los $10 \mathrm{~mm}$ y luego permanece constante. Debido a que las partículas $\beta$ virtualmente no escapan de depósitos tumorales grandes, grandes dosis de yodo 131 pueden suministrarse sin dañar los tejidos circundantes. La falta de homogeneidad en la dosis de radiación se debe a la distribución irregular del yodo 131 en los focos neoplásicos y el pequeño radio de acción de las partículas $\beta$.

La radiación $\gamma$ aporta solo $10 \%$ de radiación total y solo una pequeña fracción de rayos $\gamma$ son absorbidos por el tejido funcional, mientras que la mayor sale a través de la superficie corporal; esta radiación puede detectarse para convertirse en imágenes y es responsable de que el paciente deba permanecer aislado cuando se suministran dosis mayores a $30 \mathrm{mCi}$, conforme la legislación internacional vigente. ${ }^{6}$

La eficacia del yodo 131 es directamente proporcional a la captación y retención por el tejido tumoral. La captación tumoral efectiva ocurre si alcanza $0.5 \%$ de la dosis de yodo 131 por gramo de tejido y tiene una vida media biológica de cuatro días. Con una dosis de $150 \mathrm{mCi}$ (equivalente a $5.6 \mathrm{GBq}$ ) de yodo $131 \mathrm{el}$ tumor puede recibir 25,000 cGy, que equivale a cuatro veces la dosis liberada en una sesión de radiación de haz externo. ${ }^{7}$

\section{Biología}

El yodo es esencial para la síntesis de hormonas tiroideas. Después de su ingesta es reducido a yoduro en el intestino delgado proximal. Más de $90 \%$ es absorbido durante los primeros 60 minutos. Se distribuye en la sangre como un ion extracelular, similar al cloruro, para incorporarse a las células gracias al cotransportador $\mathrm{Na}^{+} / \mathrm{I}^{-}$(NIS). ${ }^{8}$

EI NIS es una proteína de la membrana basolateral de la célula folicular. NIS cotransporta dos iones de sodio y un ion yoduro. El gradiente de sodio provee la energía para esta transferencia y es mantenido por la bomba $\mathrm{Na}^{+} / \mathrm{K}^{+}-\mathrm{ATPasa}$. En el interior de la célula, el yodo penetra la membrana apical hasta el coloide, gracias a transportadores como la pendrina. La tiroperoxidasa (TPO) de la membrana apical actúa sobre el yodo en tres pasos secuenciales: primero lo oxida, luego lo incorpora a los residuos de tirosina de la tiroglobulina ( $\mathrm{Tg}$ ) y finalmente cataliza el acoplamiento de dos residuos de tirosina yodados para formar las futuras hormonas tiroideas. Este proceso es denominado organificación del yodo. ${ }^{9}$

Luego, dependiendo de la necesidad de hormonas tiroideas por el organismo, la Tg es endocitada por la membrana apical en forma de gotas de coloide que son degradadas por enzimas lisosomales, liberando triyodotironina y tiroxina (T3 y T4, respectivamente) al torrente sanguíneo. Este proceso es regulado por la hormona estimuladora del tiroides (TSH), que tras unirse a su receptor (TSH-r) en la membrana basolateral activa las vías dependientes del AMPc e induce el transporte de yodo por regulación de la expresión de NIS, tanto transcripcional como postranscripcional (Figura 1).

\section{Distribución normal del NIS}

Además del tejido tiroideo, se ha detectado mRNA-NIS en glándulas salivales, estómago, timo y mamas y en bajos niveles en próstata, ovario, glándulas adrenales, pulmón, y corazón. ${ }^{10}$ Esto es consistente con la captación normal del yodo 131 en glándulas salivales, estómago, mama lactante y placenta. Ocasionalmente, la absorción del yodo 131 también es observada en mamas no lactantes. ${ }^{11}$

\section{Expresión del NIS en el carcinoma tiroideo}

Usando inmunohistoquímica con anticuerpos anti-NIS se detecta NIS en menor proporción en 


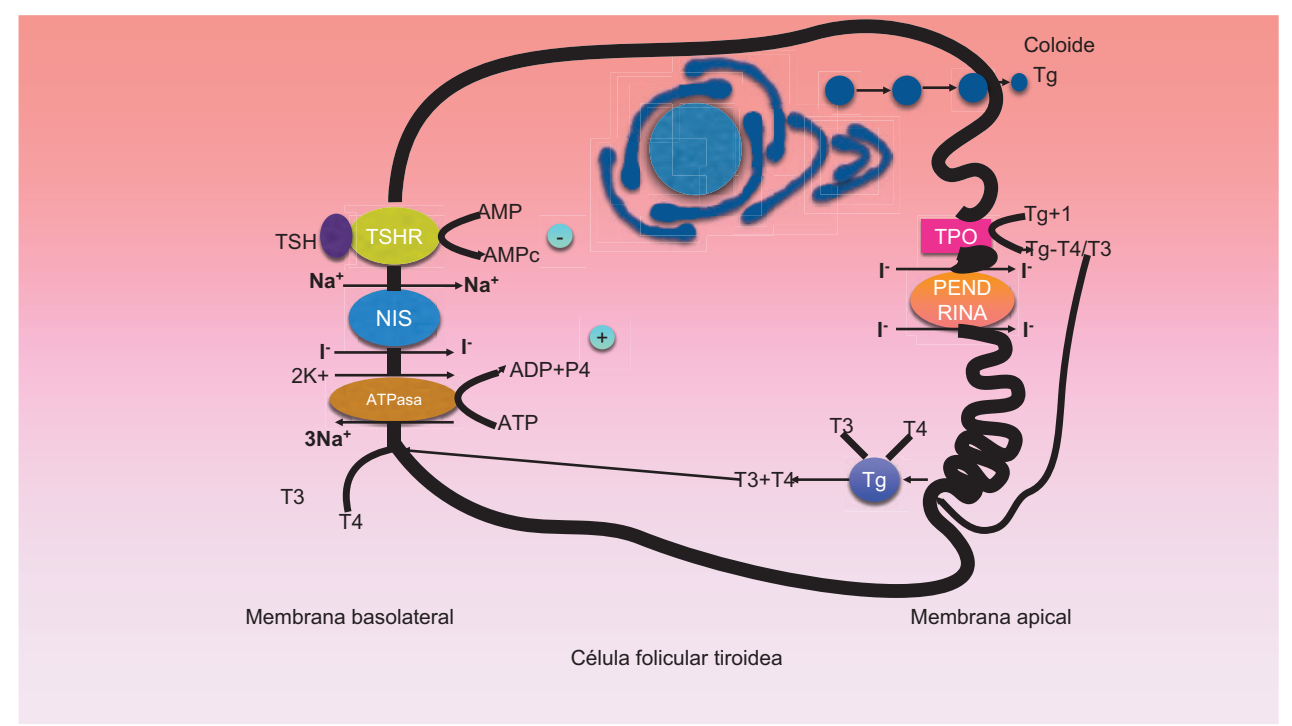

Figura 1. El cotransportador $\mathrm{Na}^{+} /$(NIS) transporta yodo junto con dos átomos de sodio de forma activa al interior de la célula por la membrana basolateral; una vez en su interior, el yodo pasa por la membrana apical al coloide gracias a la pendrina. El proceso es regulado por la hormona estimulante del tiroides.

tejidos tiroideos neoplásicos respecto los normales y se demuestra expresión heterogénea en células malignas del carcinoma papilar o folicular. En tejidos tiroideos neoplásicos, la expresión de mRNA-NIS es 10 a 1200 veces menor respecto al tejido tiroideo normal; la expresión de mRNA-TPO está reducida cinco a 500 veces, mientras que la expresión de mRNA-Tg es 300 veces menor. ${ }^{12}$ Pero Dohan et al. ${ }^{13}$ reportaron que $70 \%$ de los tumores tiroideos muestran incremento en la expresión intracelular del NIS respecto al tejido tiroideo sano. Estos resultados discordantes son explicados por diferencias metodológicas, pero también sugiere que NIS se sobreexpresa en las células neoplásicas, pero la transformación maligna interfiere con la correcta localización del NIS en la célula, lo que deteriora su función..$^{14}$

Además, la mayoría de los investigadores documenta que la expresión del NIS guarda relación inversa con la diferenciación de las células neoplásicas. NIS es expresado siempre en los cánceres más diferenciados, mientras que es negativo en la mayoría de los pobremente diferenciados..$^{15} \mathrm{La}$ localización del transportador en la membrana es indispensable para que sea funcional y capte el yodo. No basta que NIS se exprese, debe ubicarse en la membrana celular para que sea plenamente funcional. ${ }^{16}$

\section{Ablación y tratamiento con yodo 131}

La captación del yodo 131 ocurre principalmente por influencia de la TSH y la activación de AMPc, que incrementa la biosíntesis del NIS, pero otros factores también intervienen: la insulina, el factor de crecimiento insulínico tipo 1 (IGF-1) y el factor de crecimiento epidérmico (EGF).

Una medida eficaz consiste en evitar contrastes radiográficos yodados durante tres meses y cualquier medicación con alto contenido en yodo, como amiodarona, multivitamínicos, antibióticos (penicilina), jarabes para la tos (ambroxol/dextrometorfano), incluso algunos cosméticos contienen elevadas cantidades de yodo, ya que pueden interferir en la acción del yodo $131 .{ }^{17}$

\section{Estrategias para elevar la TSH}

Para estimular la captación de yodo 131 por las células tumorales usualmente se elimina la medicación supresora con levotiroxina para elevar la TSH endógena o se administra TSH recombinante humana (Thyrogen ${ }^{\circledR}$ ). Esto supone que las células neoplásicas conservan cierta diferenciación y respuesta hormonal. Ocasionalmente el cáncer de tiroides pierde esta capacidad asociándose a un pronóstico considerablemente deteriorado. ${ }^{18}$

Después de la tiroidectomía total, los niveles de T3, T4 y TSH son inicialmente normales y dos a tres semanas después los niveles de TSH comienzan a elevarse mientras que T3 y T4 disminuyen. Generalmente se evita el tratamiento sustitutivo hormonal las primeras cuatro a seis semanas desde la tiroidectomía si se ha decidido, con base en la evaluación de 
riesgos, administrar yodo 131. Sin embargo, en algunos lugares se inicia de inmediato la terapia sustitutiva hormonal. ${ }^{19}$

La elevación de la TSH por encima de $30 \mathrm{mU} / \mathrm{L}$ es necesaria para obtener la suficiente concentración de yodo 131 en las células que expresan NIS. Suspender o evitar la terapia sustitutiva hormonal por cuatro semanas en individuos tiroidectomizados abate los niveles de T3 y T4 y eleva los niveles de TSH. ${ }^{20} \mathrm{En}$ consecuencia, los pacientes se exponen a un periodo prolongado de hipotiroidismo y síntomas asociados, con un deterioro en la calidad de vida, y probabilidad de crecimiento tumoral. ${ }^{21}$

Alternativas para evitar el estado hipotiroideo causado por la suspensión de la LT4 incluyen el uso de TSH recombinante humana (rhTSH), que tiene una estructura idéntica a la TSH humana, pero su glucosilación difiere, tiene un mayor contenido de ácido siálico, lo que se asocia con menor actividad inmunológica, menor afinidad por el receptor de la TSH y menor bioactividad in vitro respecto a la TSH humana. En pacientes con CDT tiroides y función renal conservada que reciben $0.9 \mathrm{mg}$ de rhTSH tiene una vida media de 22 horas. Las concentraciones de T3 y T4 se incrementan 54 y $89 \%$, respectivamente, entre las cuatro y ocho horas subsecuentes a la administración de la rhTSH, mientras que el pico de tiroglobulina ocurre a las 48 horas. $^{22}$ En un estudio se comparó la tasa de exposición a la radiación entre individuos sometidos a supresión hormonal versus administración de rhTSH; se demostró que con la segunda, la exposición a radiación es menor y más corta, lo que resulta en menor concentración de tiroglobulina y menor captación de yodo 131 por las células tumorales. ${ }^{23,24}$ Sin embargo, la rhTSH es muy eficaz, pero relativamente costosa.

Una tercera estrategia consiste en sustituir LT4 por LT3; la segunda tiene una vida media mucho menor: uno a tres días (en lugar de siete días de la LT4), y por ello puede suspenderse por menos tiempo para elevar los niveles de TSH. ${ }^{25}$ Los pacientes que suspenden LT4 y la sustituyen por LT3 se someten solo a dos semanas de hipotiroidismo, mientras que con LT4 a cuatro.

Se ha señalado la necesidad de practicar una dieta baja en yodo dos semanas antes de la administración de yodo 131 para obtener una yoduría $<50 \mu \mathrm{g} / \mathrm{gCr} ;{ }^{26}$ con anterioridad se demostró que después de una dieta baja en yodo la captación por las células tumorales se incrementaba al doble. Ahora bien, no hay estudios que examinen si una dieta baja en yodo impacta en la tasa de recaída o mortalidad. ${ }^{27}$
La rhTSH se reserva para pacientes en los que el estado hipotiroideo puede agravar una condición médica subyacente, como síndrome metabólico mal controlado, enfermedad renal crónica (TFG $<50 \mathrm{mg} / \mathrm{mL}$ ), depresión mayor, arritmia cardiaca (en particular si el paciente está en tratamiento con amiodarona y esta es insustituible) 0 en pacientes con tejido residual o metastásico voluminoso que impida la elevación de TSH debido a la producción continua de hormonas tiroideas. ${ }^{28}$

La guía BTA sugiere que pacientes con T1-T3, N0, N1a, M0 y R0, el método de elección es administrar rhTSH; pero en pacientes de alto riesgo, en recaída o con enfermedad metastásica, el método de elección debe ser suspender LT4; no obstante, se requieren estudios prospectivos que sustenten dicha recomendación.

Independientemente de la estrategia, se debe corroborar que los niveles de TSH sean óptimos al recibir yodo 131, es decir, por encima de $30 \mathrm{mU} / \mathrm{L}$; suele medirse en conjunto con la tiroglobulina ( $\mathrm{Tg}$ ) y los anticuerpos antitiroglobulina (Ac-antiTg) (Figura 2). ${ }^{29}$

\section{Evaluación de riesgo}

Aún existen controversias relativas a la ablación con RAI, pero se aduce que administrar yodo 131 simplifica el seguimiento al eliminar el tejido tiroideo normal o neoplásico productor de $\mathrm{Tg}$, lo que facilita la identificación de recaídas midiendo la $\mathrm{Tg}$ y evitando la producción continua de anticuerpos anti-Tg (Ac-antiTg). ${ }^{30}$ La decisión de suministrar dosis ablativas debe ser racional, basada en evaluaciones del riesgo. Las guías de la American Thyroid Association (ATA 2015) y la British Thyroid Association (BTA, 2014) proponen sistemas similares, incluso la National Comprehensive Cancer Network (NCCN V1.2016) concuerda en varios puntos (Tabla 1). ${ }^{31,32}$

La guía ATA surgió de la incapacidad de otros sistemas para establecer el riesgo de recaída; los sistemas previos solo son útiles para establecer el riesgo de mortalidad. Es un hecho que un paciente con bajo riesgo de mortalidad puede tener alto riesgo de recaída, por tal motivo, ATA, BTA y NCCN recomiendan realizar la evaluación del riesgo de recaída en todos los pacientes.

\section{¿Quiénes deben recibir yodo 131 y cuál es la dosis apropiada?}

La finalidad de administrar yodo 131 es eliminar cualquier depósito microscópico de carcinoma tiroideo y 


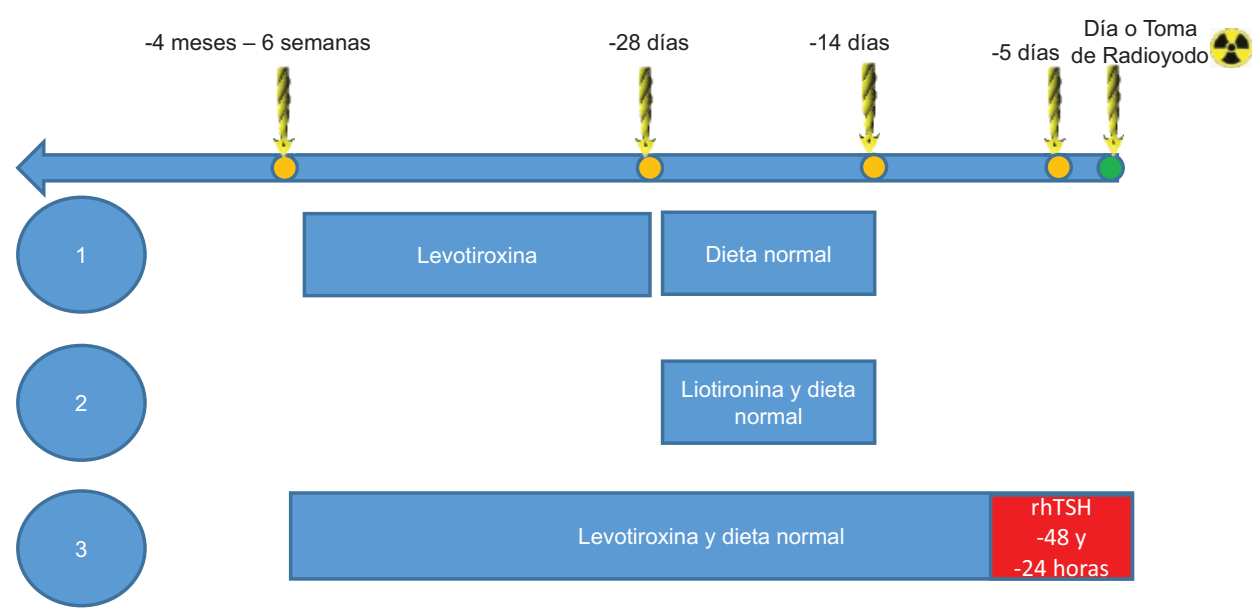

Figura 2. Estrategias para elevar la hormona estimulante del tiroides. Para lograr niveles óptimos para recibir yodo 131 se pueden seguir tres estrategias: suspender la levotiroxina cuatro semanas antes de la administración de yodo 131, intercambiar levotiroxina por liotironina, la cual se suspende dos semanas antes del yodo 131, y optar por administrar rhTSH sin suspender hormonas tiroideas.

Tabla 1. Grupos de riesgo para cáncer de tiroides de acuerdo con la American Thyroid Association

\begin{tabular}{lll}
\hline Riesgo bajo & Riesgo intermedio & Riesgo alto \\
\hline Todos los siguientes están presentes: & Alguno de los siguientes está presente: & Alguno de los siguientes está presente: \\
- Sin metástasis. & - Extensión extratiroidea microscópica. & - Extensión extratiroidea macroscópica. \\
- Resección completa. & - Captación de yodo 131 fuera del lecho & - Resección incompleta del tumor (R2). \\
- Sin extensión extratiroidea. & tiroideo luego de ablación de remanentes. & - Metástasis. \\
- Sin histología agresiva. & - Histología agresiva (células altas, insular, & - Niveles elevados de Tg poscirugía. \\
- Sin invasión vascular. & columnares, en tachuela, células de & - N1 patológico con cualquier número de \\
- Sin captación fuera del lecho tiroideo & Hulte). & ganglios metastásicos o dimensión global \\
posablación con yodo 131. & - Carcinoma papilar con invasión vascular. & mayor a 3 cm. \\
- NO, < 5 N1 micrometástasis. & - N1 patológico $(>5$ ganglios patológicos) & - Carcinoma folicular con invasión vascular \\
- Variante folicular encapsulada, intratiroidea. & con dimensión global no mayor a 3 cm. & extensa (> 4 focos). \\
- Carcinoma folicular bien diferenciado, & - Carcinoma papilar multifocal con & \\
intratiroideo con invasión capsular mínima & extensión extratiroidea mínima y mutación & \\
y sin o con mínima invasión vascular $(<4$ & de BRAF V600E. & \\
focos). & & \\
- Microcarcinoma papilar intratiroideo, uní o & & \\
multifocal, sin mutación de BRAF V600E. & & \\
\hline
\end{tabular}

tejido tiroideo normal. NCCN y ATA proponen tres objetivos:

- Ablación del tejido tiroideo remanente (el término ablación solo debe emplearse cuando se administra la primera dosis de yodo 131 para simplificar el seguimiento).

- Terapia adyuvante, para eliminar micrometástasis subclínicas.

- Dosis terapéutica para tratar la persistencia local o metástasis confirmadas.

Sin embargo, para fines prácticos, la aplicación de yodo 131 suele clasificarse en ablación y tratamiento. La eficiencia del yodo 131 es inversamente proporcional al volumen del tejido tiroideo residual y directamente proporcional a los niveles de TSH, por lo que debe eliminarse quirúrgicamente toda la enfermedad macroscópica. $^{33}$
A la fecha existen tres enfoques para definir la dosis de yodo 131: dosis fija, dosis determinada por el límite superior de la sangre y dosimetría tumoral cuantitativa, los dos últimos a menudo se reservan para pacientes con metástasis o situaciones inusuales, como insuficiencia renal, niños, ancianos y aquellos con extensas metástasis pulmonares. Debido a su complejidad, se favorece la estrategia de dosis fijas. ${ }^{34}$

\section{Pacientes con riesgo bajo}

Estudios prospectivos del Nacional Thyroid Cancer Treatment Cooperative Study Group (NTCTCSG) sugieren que la supervivencia libre de progresión y la supervivencia global enfermedad específica no mejoran con el tratamiento con yodo 131 en pacientes estadio I o II. Un estudio retrospectivo multicéntrico, 
donde se evaluaron 1298 pacientes de bajo riesgo, con seguimiento promedio de 10.3 años, no encontró beneficios de la ablación con yodo 131 en relación con la supervivencia global y la supervivencia libre de progresión. ${ }^{35}$ En concreto, existe poca evidencia que apoye el uso de yodo 131 en estos pacientes.

Aunque no existen ventajas en términos de supervivencia, ciertos estudios han demostrado la conveniencia de dosis bajas versus altas en pacientes con bajo riesgo, independientemente del método de estimulación. ${ }^{36}$ Cheng et al. ${ }^{37}$ analizaron nueve estudios aleatorizados, incluyendo 2569 pacientes de bajo riesgo y diferentes métodos de estimulación de TSH (rhTSH y suspensión hormonal). No encontró diferencias en la eficacia del yodo 131 entre $30 \mathrm{mCi}$ y $100 \mathrm{mCi}$.

La ablación con 30 versus $100 \mathrm{mCi}$ es equivalente, independientemente del método de estimulación; la ventaja de dosis no mayores de $30 \mathrm{mCi}$ estriba en que los pacientes no requieren hospitalización, existe calidad de vida similar y los efectos secundarios relacionados con el yodo 131, como sialoadenitis, edema, náusea y vómito son menores; además, existe una menor tasa de exposición a radiación y menor dosis acumulada. Recientes análisis y revisiones sistemáticas apoyan están conclusiones. ${ }^{38}$

\section{Pacientes con riesgo intermedio}

Respecto a esta categoría existen discrepancias en la literatura. ${ }^{39} \mathrm{La}$ administración de yodo 131 en pacientes con tumores histológicamente agresivos (células altas, esclerosante difusa, etcétera) mejora la supervivencia global, sin embargo, en estudios con pacientes con ganglios positivos, extensión extratiroidea microscópica o tumor $>4 \mathrm{~cm}$, la supervivencia es similar si los pacientes son menores de 45 años, aunque el tiempo de seguimiento no superó los siete años. Por otro lado, existe ventaja en los individuos $>45$ años que recibieron tratamiento; aunque no superó $73 \%$, fue superior a $69 \%$ del grupo que no la recibió. ${ }^{40}$-Lamartina et al. ${ }^{41}$ en la revisión de 13 estudios no aleatorizados no encontraron beneficios, mientras que 11 apoyaban su uso. Consideramos que se requieren más estudios, con criterios estandarizados y homogéneos.

\section{Pacientes con riesgo alto}

Se recomienda la ablación rutinaria en pacientes con alto riesgo y se sugiere que la primera dosis de yodo 131 no supere los $150 \mathrm{mCi}$, en especial en individuos $>45$ años. ${ }^{42}$ Para el tratamiento de la recaída o persistencia se han propuesto diferentes enfoques. Se usaba el concepto de dosis fijas altas, es decir 100-150 mCi si la persistencia o recaída estaba confinada al lecho tiroideo; 150-175 mCi si hubo afección de ganglios linfáticos, 200-250 mCi si existían metástasis en pulmones; en caso de metástasis óseas se recurría a 200-300 $\mathrm{mCi}^{43}$ Sin embargo, la eficacia del yodo 131 está relacionada con la dosis de radiación liberada al tejido neoplásico y la radiosensibilidad del mismo. De manera que, la radiosensibilidad resulta mayor en jóvenes (< 45 años), con pequeñas metástasis $(<10 \mathrm{~mm})$, tumores bien diferenciados (papilar y folicular) y ávidos por yodo 131.

Actualmente se reconoce que la dosis de radiación absorbida máxima tolerada (MTRD, por sus siglas en inglés) es de 200 cGy en sangre ${ }^{44}$ y a mayor edad la función renal disminuye, como sucede en pacientes con insuficiencia renal o falla cardiaca, y con ello el aclaramiento del yodo 131 disminuye..$^{45}$ En este sentido, Tuttle et al. ${ }^{46}$ demostraron que dosis empíricas de $140 \mathrm{mCi}$ excedían la MTRD en menos de $7 \%$ de los pacientes < 70 años; este porcentaje se incrementaba a $13 \%$ en > 80 años; por otra parte, la dosis de 200 y $250 \mathrm{mCi}$ excedían la MTRD en < 70 años en 15 y $22 \%$, respectivamente, mientras que en $>70$ años hasta en 38 y $50 \%$, respectivamente. Es posible administrar dosis $>200 \mathrm{mCi}$ siempre y cuando se realice un estudio dosimétrico previo y los pacientes sean $<65$ años.

Actualmente no se recomienda administrar yodo 131 en dosis $>200 \mathrm{mCi}$, ya que no se ha demostrado su superioridad, por el contrario, la exposición a radiación es mayor, los efectos secundarios más frecuentes y prolongados y la dosis acumulada es mayor sin mejor supervivencia.

\section{Utilidad del rastreo pre y postratamiento con yodo 131}

Algunos centros de medicina nuclear realizan un rastreo de cuerpo completo antes de la dosis ablativa o terapéutica, para estimar la dosis de yodo 131 necesaria. Con la disponibilidad de equipos de SPECT/ CT se ha incrementado la sensibilidad y especificidad respecto al rastreo planar. El rastreo planar alcanza una sensibilidad de $41 \%$ y especificidad de $68 \%$; mientras que la sensibilidad del SPECT es de $45 \%$ y la especificidad de $89 \%$. Con el SPECT/CT, la 
sensibilidad se incrementa hasta $50 \%$ y la especificidad alcanza $100 \%{ }^{47}$

Avram et al., en una serie de 320 pacientes a los que practicó SPECT/CT preablación con yodo 131, reestadificaron a $4 \%$ de los pacientes $<45$ años y a $25 \%$ de los $>45$ años. ${ }^{48}$ Con el uso generalizado de factores clínico-patológicos, actualmente no se recomienda rastreo preablación como estrategia para definir la dosis, sin embargo, puede ser necesario en pacientes en los que la enfermedad residual no ha sido evaluada con un estudio histopatológico o mediante ultrasonido.

El "aturdimiento" es definido como una disminución en la captación del yodo 131 por células tiroideas neoplásicas después de la administración de una dosis diagnóstica de yodo $131 .{ }^{49}$ Puede deberse a la reducción en el número de células tiroideas funcionales debido a muerte celular producida por la radiación $\beta$ del yodo 131 o por disminución de la capacidad de las células tiroideas viables para atrapar o retener yodo 131 durante un intervalo de tiempo. Guiraud-Vitaux et al. ${ }^{50}$ señalan que puede existir necrosis infraterapéutica debido a altas dosis de yodo 131, lo cual implicaría una reducción de la eficacia de las dosis terapéuticas tras el diagnóstico por imagen. Sin embargo, la investigación de este fenómeno ha conducido a conclusiones contradictorias. ${ }^{51}$ Investigaciones recientes han demostrado que es un fenómeno radiobiológico estrechamente relacionado con la radiación absorbida, más que con la dosis administrada, y que el aturdimiento a menudo se observa 48 horas después de una dosis de yodo $131 .{ }^{52}$

En otro orden de ideas, se recomienda practicar un rastreo corporal cinco a ocho días después de la ablación o dosis terapéutica, ya que identifica focos metastásicos adicionales en 10 a $26 \%$ de los pacientes cuando se le compara con un rastreo diagnóstico. EI SPECT/CT mejora la sensibilidad hasta $78 \%$ y la especificidad hasta $100 \%$, reduciendo la necesidad de estudios seccionales en $20 \%$ (Figura 3). ${ }^{53}$

\section{El seguimiento}

El rastreo de cuerpo completo más SPECT/CT con yodo 131 es usado en el seguimiento, al menos seis meses después de la dosis para ablación o terapéutica según la ATA o nueve según la BTA, bajo tres escenarios: pacientes con captación anormal fuera del lecho tiroideo después del rastreo postratamiento o posablación; en pacientes con escasa información en el rastreo posablación debido a grandes remanentes que impiden definir si existió captación fuera del lecho tiroideo; y en pacientes con presencia o aparición de novo de Ac-antiTg, incluso en ausencia de hallazgos sospechosos de enfermedad residual por ultrasonido.

Deben conjuntarse hallazgos clínicos, bioquímicos y de imagen durante el seguimiento para redefinir el tratamiento, otorgar la terapia más apropiada y definir la respuesta. Tuttle et al. ${ }^{54} \mathrm{y}$ Vaisman et $a l . .^{55}$ han contribuido a una mejor evaluación, definiendo cuatro categorías de respuesta:

- Respuesta excelente, se obtiene cuando no existe evidencia de enfermedad estructural (por imagen con ultrasonido o SPECT/CT) y clínica o bioquímica ( $\mathrm{Tg}$ suprimida $<0.2 \mathrm{ng} / \mathrm{mL}$ o estimulada $<1 \mathrm{ng} / \mathrm{mL}$ ). Ocurre en 86 a $91 \%$ de los pacientes con bajo riesgo, en 57 a $63 \%$ de los pacientes con riesgo intermedio y 14 a $16 \%$ con riesgo alto.

- Respuesta bioquímica incompleta, resulta cuando existe un método de imagen negativo, pero niveles de Tg suprimida $>1 \mathrm{ng} / \mathrm{mL}, \mathrm{Tg}$ estimulada $>10 \mathrm{ng} / \mathrm{mL}$ o elevación de los Ac-antiTg. Esta respuesta la presentan 11 a $19 \%$ de los pacientes de bajo riesgo, 21 a $22 \%$ de aquellos con riesgo intermedio y 16 a $18 \%$ con riesgo alto.

- Respuesta estructural incompleta, se refiere a los pacientes con enfermedad estructural o funcional por cualquier método de imagen, con cualquier nivel de $\mathrm{Tg} \circ \mathrm{Ac}$-antiTg, la cual está presente en 2 a $6 \%$ de los pacientes con bajo riesgo, 19 a $28 \%$ con riesgo intermedio y 67 a $75 \%$ de aquellos con riesgo alto.

- Respuesta indeterminada, se refiere a hallazgos inespecíficos en estudios de imagen, niveles de $\mathrm{Tg}$ suprimida $<1 \mathrm{ng} / \mathrm{mL}$, niveles de Tg estimulada $<10 \mathrm{ng} / \mathrm{mL}$ o niveles estables de Ac-antiTg. La respuesta está presente en 12 a $29 \%$ de los pacientes con bajo riesgo, 8 a $23 \%$ con riesgo intermedio y 0 a $4 \%$ con riesgo alto.

No es suficiente realizar rastreos de cuerpo completo con SPECT/CT, debe correlacionarse con los marcadores específicos, $\mathrm{Tg}$ y anticuerpos-anti $\mathrm{Tg}$. Es bien conocido que las mediciones de los niveles de $\mathrm{Tg}$ son de particular importancia para el monitoreo de los pacientes con enfermedad residual y recaída. En ausencia de Ac-anti Tg, la Tg tiene elevada sensibilidad y especificidad para documentar recaída o persistencia de la enfermedad. Numerosos autores recomiendan su medición con la TSH estimulada debido a que puede incrementar los niveles de Tg entre cinco y 10 veces. Los niveles de Tg estimulada $<1 \mathrm{ng} /$ 


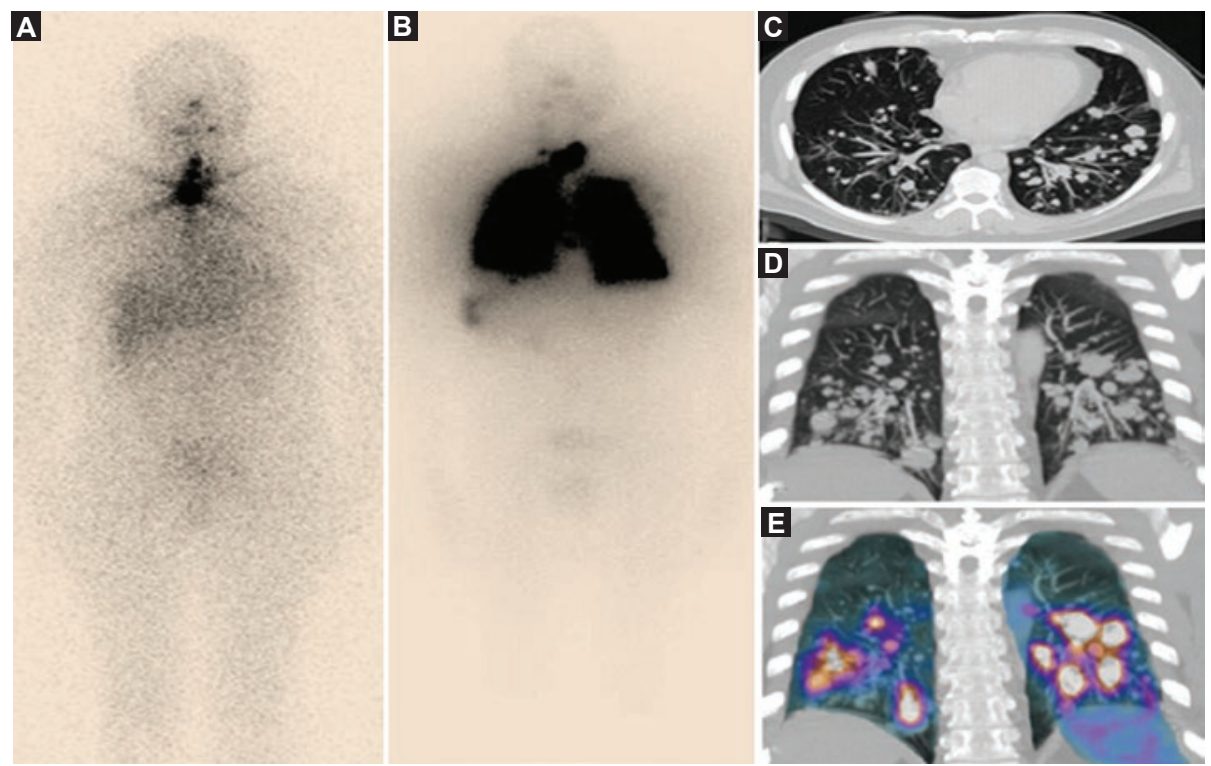

Figura 3. (A) Rastreo planar en proyección anterior practicado 48 horas después de administrar 5 mCi de yodo 131. Se observa intensa captación focal anormal limitada al lecho tiroideo. Debido a la sintomatología respiratoria se practicó una tomografía computarizada sin contraste, en la que se observaron múltiples imágenes nodulares en ambos pulmones compatibles con metástasis pulmonares (C y D), por ello se decidió administrar $200 \mathrm{mCi}$ de yodo 131; a los siete días, en el rastreo planar en proyección anterior se observó intensa captación en lecho tiroideo en ambos pulmones (B). Posterior al rastreo, un SPECT/CT evidenció infiltrado pulmonar y captación del yodo 131, no visualizado en el rastreo planar preablación (E).

$\mathrm{mL}$ o la ausencia de anticuerpos-anti Tg tienen probabilidad de $98 \%$ para identificar pacientes con ausencia de la enfermedad. ${ }^{56}$

Por otro lado, los Ac-anti-Tg pueden estar presentes o aparecer de novo hasta en $25 \%$ de los pacientes, en particular en aquellos con tiroiditis de Hashimoto. ${ }^{57}$ Las enfermedades autoinmunes tiroideas se asocian con producción de anticuerpos en los linfocitos intratiroideos y después de una tiroidectomía total los niveles permanecen por años elevados sin evidencia de enfermedad. La razón es que en los ganglios linfáticos cervicales se inicia y disemina la respuesta autoinmune en respuesta a la de $\mathrm{Tg}$ en las células presentadoras de antígeno. La vida media de los Ac-anti-Tg después de una tiroidectomía total es de 10 semanas, decremento rápido que se debe a la formación de complejos Tg-anticuerpos anti-Tg formados en respuesta a la elevación de la $\mathrm{Tg}$ después de la cirugía. ${ }^{58}$

La cirugía puede iniciar o propiciar aparición de novo de Ac-antiTg, las cuales tienden a declinar en el curso de meses. Los pacientes pueden no alcanzar un estado negativo de Ac-antiTg durante el primer año posoperatorio e incluso exhibir aumento (o la aparición de novo) durante los seis meses posteriores al tratamiento con yodo 131 cuando hay liberación de $\mathrm{Tg}$ secundaria a daño radiolítico del tejido tiroideo. ${ }^{59}$

Para un seguimiento eficaz de los Ac-anti-Tg a largo plazo es fundamental utilizar un método sensible cada seis a 12 meses. Kim et al. ${ }^{60}$ encontraron que en pacientes con descenso mayor a $50 \%$ de los niveles de Ac-antiTg entre los seis y 12 meses posteriores al yodo $131,<1 \%$ desarrollaba recaída. En contraste, $19 \%$ de pacientes con descenso menor de $50 \%$ en el mismo intervalo presentaba recaída, mientras que $37 \%$ en los que incrementaron niveles presentaron recaída.

\section{El rol del PET/CT con 2-[18F]-fluoro-2- deoxy-D-glucosa (PET/CT-FDG)}

EI PET/CT-FDG es una valiosa herramienta en pacientes con elevación de los niveles de $\mathrm{Tg}$ o anticuerpos-Anti-Tg y rastreo con yodo 131 negativo. ${ }^{61}$ En neoplasias tiroideas, la captación del radiofármaco 18F-FDG se limita a tumores más agresivos o de alto grado, con nula o escasa captación por tumores bien diferenciados, fenómeno al que Feine et al. ${ }^{62}$ denominó flip-flop.

Debido a su carácter hidrófilo, la glucosa requiere GLUT (proteínas transmembrana trasportadoras de glucosa) que le permita cruzar la membrana celular. La sobreexpresión de GLUT1 en la membrana celular de las neoplasias tiroideas está estrechamente relacionada con tumores con comportamiento biológico más agresivo. Además, la TSH desempeña un rol importante ya que estimula el transporte de glucosa y la actividad glucolítica en los tirocitos mediante la translocación de GLUT1, así como la neosíntesis de GLUT1 por la 


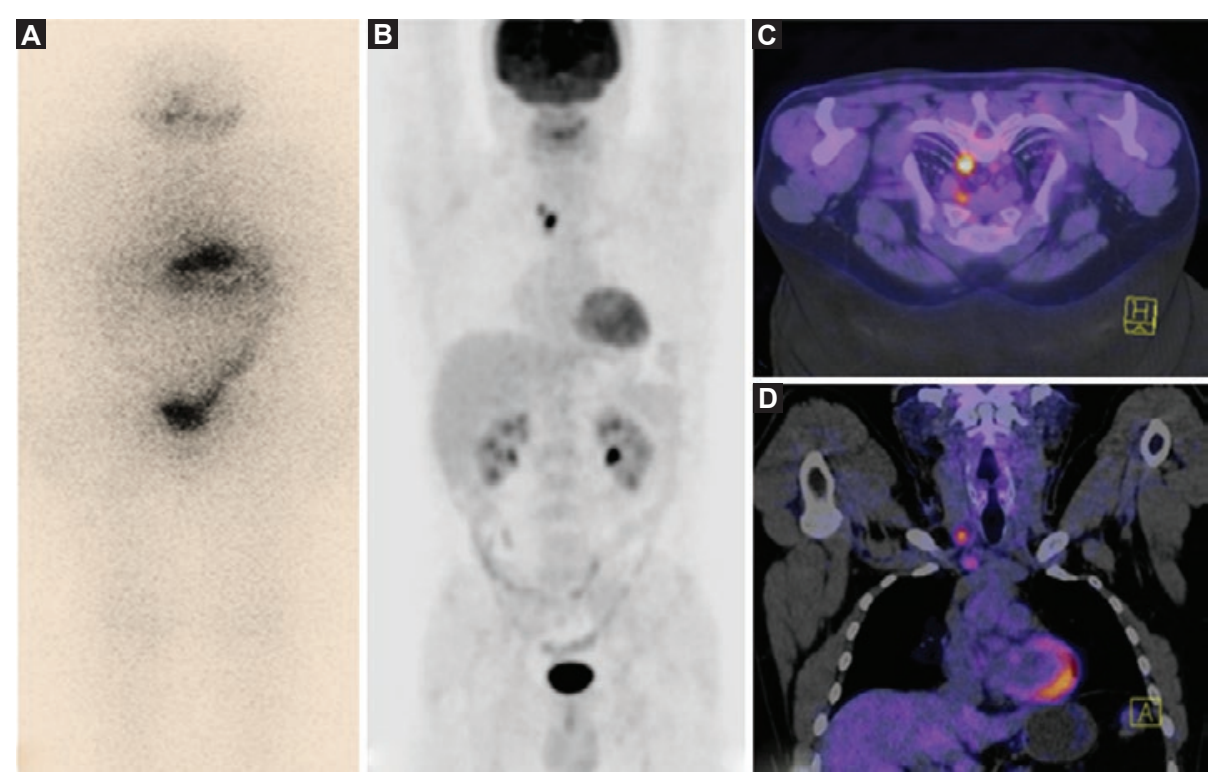

Figura 4. (A) Rastreo planar en proyección anterior realizado 48 horas después de la administración de 5 mCi de yodo 131. Se observó biodistribución y vías de eliminación habituales, sin zonas focales de captación anormal en un paciente con TSH estimulada y Tg > $100 \mathrm{ng} / \mathrm{mL}$. (B) Siete días después se practicó un PET/CT-FDG, con el que se observó captación focal anormal en la línea media paraesternal derecha. (C y D) En cortes axiales y coronales se apreciaron dos nódulos con intensa captación del radiotrazador en ganglios cervicales nivel VI derechos y en ganglios mediastinales niveles $1 R$ y $3 A$.

activación de su expresión génica, lo que indica que la sensibilidad del PET/CT-FDG es influida por los niveles de la TSH. ${ }^{63}$

Salvatori et al. ${ }^{64}$ sugieren que las indicaciones para practicar PET/CT-FDG se dividan en fuertemente definidas y no completamente definidas. En las primeras se deberán incluir pacientes con niveles elevados de $\mathrm{Tg}$ (> $10 \mathrm{ng} / \mathrm{mL}$ ) y estudio de imagen negativo (ultrasonido, rastreo con yodo 131). La elevación de Ac-anti $\mathrm{Tg}$ o su aparición de novo puede alterar drásticamente los valores de la $\mathrm{Tg}$, por lo tanto, también es indicación la elevación de Ac-anti $\mathrm{Tg} 0$ aparición de novo ante niveles de $\mathrm{Tg}<2 \mathrm{ng} / \mathrm{mL}$.

La importancia pronóstica de PET/CT-FDG radica en que la mayor parte de los pacientes con enfermedad metastásica tiene avidez por el 18F-FDG, lo que sugiere mayor agresividad, desdiferenciación y células metabólicamente activas; un PET/CT-FDG negativo predice pronóstico favorable. ${ }^{65} \mathrm{En}$ un análisis multivariado, Wang et al. ${ }^{66}$ analizaron 400 pacientes con características de alto riesgo; observaron que la edad y un PET/ CT-FDG son los factores pronóstico más importantes, ya que hay relación inversa entre supervivencia y el número de lesiones metabólicamente activas.

La sensibilidad y especificidad guarda relación con los niveles de Tg. Un metaanálisis, Leboulleux ${ }^{67}$ demostró que la sensibilidad y especificidad está fuertemente influida por un punto de corte de $\mathrm{Tg}>10 \mathrm{ng} / \mathrm{mL}$, siendo de 83 y $84 \%$, respectivamente, en pacientes con enfermedad no yodocaptante. Otro análisis demostró que la sensibilidad a niveles de $\mathrm{Tg}<10 \mathrm{ng} / \mathrm{mL}$, $>10$ pero $<100 \mathrm{ng} / \mathrm{mL} y>100 \mathrm{ng} / \mathrm{mL}$ fue de $10.5,75.6$ y $91 \%$, respectivamente. Además, la sensibilidad también depende del lugar y tamaño de las metástasis. Para metástasis ganglionares $<10 \mathrm{~mm}$ la sensibilidad es de $69.7 \%$ y la especificidad de $83.3 \%$, mientras que para $>11 \mathrm{~mm}$ la sensibilidad fue de $95.7 \%$ y la especificidad de $80 \%$. Por otro lado, con metástasis pulmonares < $10 \mathrm{~mm}$ la sensibilidad fue de $41 \%$ y la especificidad de $80 \%$; para $>11 \mathrm{~mm}$ la sensibilidad fue de $94 \%$ y la especificidad de $89 \% .{ }^{68}$

Como mencionamos, la sensibilidad del SPECT/CT es mayor, por lo que la ausencia de hallazgos con niveles elevados de Tg o Ac-anti Tg también indica la necesidad de PET/CT-FDG (Figura 4).

Debido a la reciente implementación en algunos centros acerca de las mutaciones oncogénicas relacionadas con el BRAF (V600E), existen algunos estudios que demuestran la existencia de una correlación entre el hipermetabolismo en los pacientes con dicha mutación. ${ }^{69}$ Nagarajah et al. ${ }^{70}$ demostraron que de una muestra de 48 pacientes con CDT, 24 presentaron mutación de BRAF (V600E) y 24, de BRAF-wild type; observaron una correlación significativamente mayor de la actividad metabólica en los pacientes con mutación del BRAF (V600E). Concluyendo que una tasa metabólica alta puede estar en relación con mutación del gen, lo que conlleva a mayor agresividad tumoral 


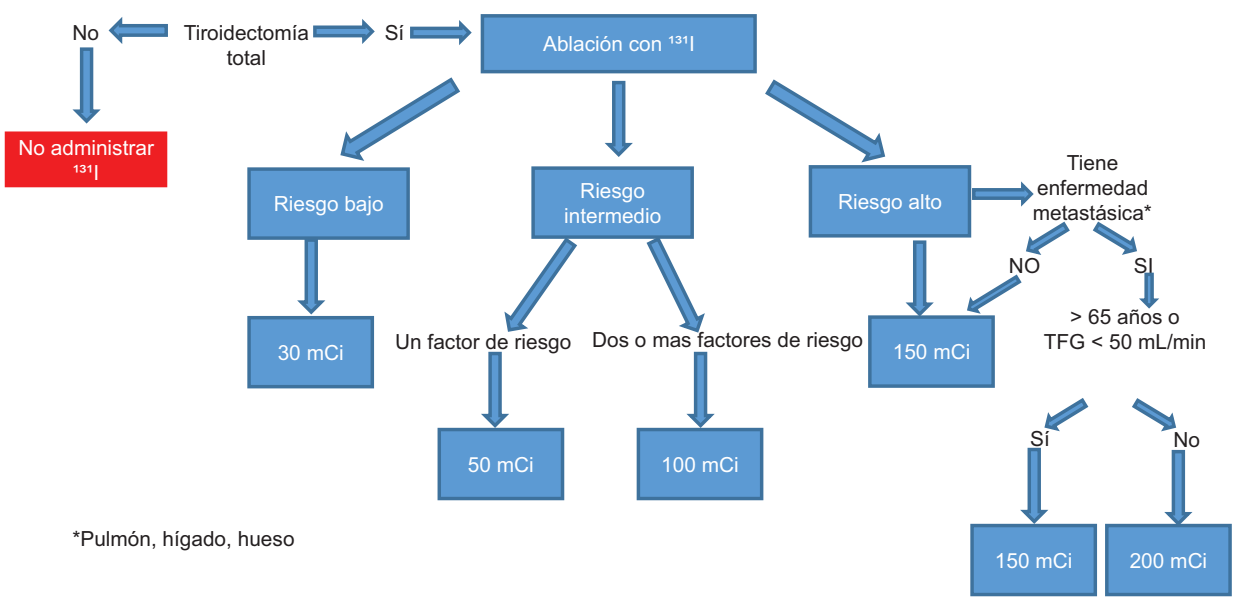

Figura 5. Recomendaciones según escenario clínico posterior a la administración de radioyodo, con base en la literatura revisada y sin unificación de criterios establecidos para grupos de riesgo intermedio y alto.

y peor pronóstico, sin embargo, se necesitan más estudios para correlacionar estos hallazgos.

Entre las indicaciones no completamente definidas se incluye la práctica de PET CT en pacientes con enfermedad refractaria, para evaluar la respuesta al tratamiento con terapias blanco, en la estadificación y seguimiento de histologías de alto riesgo, en nódulos tiroideos indeterminados después de biopsia por aspiración y recientemente para cirugía radioguiada. ${ }^{71}$

La Figura 5 ilustrar el seguimiento y tratamiento de los pacientes con CDT manejados con yodo 131.

\section{Agradecimientos}

A las doctoras Claudia Mosqueda y Angélica Arellano, por su colaboración en la generación y edición de las ilustraciones.

\section{Bibliografía}

1. Ferlay J, Soerjomataram I, Ervik M, Dikshit R, Eser S, Mathers C, et al. GLOBOCAN 2012 v1.0, Cancer incidence and mortality worldwide: IARC Cance Base No. 11. Lyon, France: International Agency for Research on Cancer; 2013. Disponible en: http://globocan.iarc.fr, accessed on 20/December/2016

2. Bongiovanni M, Paone G, Ceriani L, Pusztaszeri M. Cellular and molecular basis for thyroid cancer imaging in nuclear medicine. Clin Trans Imaging. 2013;1:149-161.

3. Granados-García M, León-Takahashi AM, Guerrero-Huerta FJ, Taissoun-Aslan ZA. Cáncer diferenciado de tiroides: una antigua enfermedad con nuevos conocimientos. Gac Med Mex. 2014;150:65-77.

4. Robbins R, Schulemberg M. The evolving role of 1311 for the treatment of differentiated thyroid carcinoma. J Nucl Med. 2005;46:28S-37S.

5. Maxon HR, Thomas SR, Hertzberg VS, Kereiakes JG, Chen IW, Sperling MI, et al. Relation between effective radiation dose and outcome of radioiodine therapy for thyroid cancer. N Engl J Med. 1983;309:937-941.

6. Braunstein GD (editor). Thyroid Cancer. Volume 32 of Endocrine Updates. EE.UU.: Springer Science \& Business Media; 2011.

7. Dorn R, Kopp J, Vogt H, Heidenreich P, Carroll RG, Gulec SA. Dosimetry-guided radiactive iodine treatment in patients with metastatic differentiated thyroid cancer. Largest safe dose using a risk-adapted approach. J Nucl Med. 2003;44:451-456.

8. Eskandari S, Loo DD, Dai G, Levy O, Wright EM, Carrasco N. Thyroid $\mathrm{Na}+/$ l-symporter. Mechanism, stoichiometry, and specificity. J Biol Chem. $1997 ; 272: 27230-27238$
9. De-La-Vieja A, Dohan O, Levy O, Carrasco N. Molecular analysis of the sodium/iodide symporter: impact on thyroid and extrathyroid pathophysiology. Physiol Rev. 2000;80:1083-1105.

10. Caillou B, Troalen F, Baudin E, Talbot M, Filetti S, Schlumberger M, et al. $\mathrm{Na}+/ \mathrm{l}$ - symporter distribution in human thyroid tissues: an immunohistochemical study. J Clin Endocrinol Metab. 1998;83:4102-4106.

11. Daniels GH, Haber DA. Will radioiodine be useful in treatment of breast cancer? Nat Med. 2000;6:859-860.

12. Filetti S, Bidart JM, Arturi F, Caillou B, Russo D, Schlumberger M. Sodium/iodide symporter: a key transport system in thyroid cancer cell metabolism. Eur J Endocrinol. 1999;141:443-457.

13. Dohán O, Baloch Z, Banrevi Z, Livolsi V, Carrasco N. Predominant intracellular overexpression of the $\mathrm{Na}+/ \mathrm{l}+$ symporter (NIS) in a large sampling of thyroid cancer cases. J Clin Endocrinol Metab. 2001;86:26972700.

14. Saito T, Endo T, Kawaguchi A, Ikeda M, Katoh R, Kawaoi A, et al. Increased expression of the sodium/iodide symporter in papillary thyroid carcinomas. J Clin Invest. 1998;101:1296-1300.

15. Min JJ, Chung JK, Lee YJ, Jeong JM, Lee DS, Jang JJ, et al. Relationship between differentiation and expression of sodium/iodide symporter or glucose transporter-1 in differentiated thyroid carcinoma. J Nucl Med. 2001:42:133P

16. Wapnir IL, Van-De-Rijn M, Nowels K, Amenta PS, Walton K, Montgomery $\mathrm{K}$, et al. Immunohistochemical profile of the sodium/iodide symporter in thyroid, breast, and other carcinomas using high-density tissue microarrays and conventional sections. J Clin Endocrinol Metab. 2003;88:1880-1888.

17. Sawka AM, Ibrahim-Zada I, Galacgac P, Tsang RW, Brierley JD, Ezzat S et al. Dietary iodine restriction in preparation for radiactive iodine treatment or scanning in well-differentiated thyroid cancer: a systematic review. Thyroid. 2010;20:1129-1138.

18. Thyroid cancer. En: Clinical Practice Guidelines in Oncology. EE.UU.: National Comprehensive Cancer Network.

19. Haugen BR, Alexander EK, Bible KC, Doherty GM, Mandel SJ, Nikiforov YE, et al. 2015 American Thyroid Association Management Guidelines for Adult Patients with Thyroid Nodules and Differentiated Thyroid Cancer: The American Thyroid Association Guidelines Task Force on Thyroid Nodules and Differentiated Thyroid Cancer. Thyroid. 2016:26:1-133.

20. Golger A, Fridman TR, Eski S, Witterick IJ, Freeman JL, Walfish PG. Three-week thyroxine withdrawal thyroglobulin stimulation screening test to detect low-risk residual/recurrent well-differentiated thyroid carcinoma. J Endocrinol Invest. 2003;26:1023-1031.

21. Dow KH, Ferrell BR, Anello C. Quality-of-life changes in patients with thyroid cancer after withdrawal of thyroid hormone therapy. Thyroid. 1997:7:613-619.

22. Almeda-Valdés $P$, Arechavaleta-Granell $M$, Bolaños-Gil-De-Montes $F$, Cuevas-Ramos D, Gamboa-Domínguez A, Gómez-Cruz R, et al. Guía clínica para el diagnóstico y tratamiento del cáncer diferenciado de tiroides. México: Sociedad Mexicana de Nutrición y Endocrinología; 2009.

23. Pötzi C, Moameni A, Karanikas G, Preitfellner J, Becherer A, Pirich C, et al. Comparison of iodine uptake in tumour and nontumour tissue under thyroid hormone deprivation and with recombinant human thyrotropin in thyroid cancer patients. Clin Endocrinol (Oxf). 2006;65:519-523.

24. Rosário PW, Borges MA, Purisch S. Preparation with recombinant human thyroid-stimulating hormone for thyroid remnant ablation with $131 \mathrm{l}$ is associated with lowered radiotoxicity. J Nucl Med. 2008;49:1776-1782. 
25. Leboeuf R, Perron P, Carpentier AC, Verreault J, Langlois MF. L-T3 preparation for whole-body scintigraphy: a randomized-controlled trial. Clin Endocrinol (Oxf). 2007;67:839-844

26. Pluijmen MJ, Eustatia-Rutten C, Goslings BM, Stokkel MP, Arias AM, Diamant $\mathrm{M}$, et al. Effects of low-iodide diet on postsurgical radioiodide ablation therapy in patients with differentiated thyroid carcinoma. Clin Endocrinol (Oxf). 2003;58:428-435.

27. Sonenberg M. Low-iodine diet in the treatment of differentiated thyroid cancer with radiactive iodine. Endocrine. 2002;17:141-143.

28. Robbins RJ, Driedger A, Magner J. Recombinant human thyrotropin-assisted radioiodine therapy for patients with metastatic thyroid cancer who could not elevate endogenous thyrotropin or be withdrawn from thyroxine. Thyroid. 2006;16:1121-1130.

29. Liel Y. Preparation for radiactive iodine administration in differentiated thyroid cancer patients. Clin Endocrinol (Oxf). 2002;57:523-527.

30. Van-Nostrand D, Wartofsky L. Radioiodine in the treatment of thyroid cancer. Endocrinol Metab Clin North Am. 2007;36:807-822.

31. Perris P, Colley S, Boelaert K, Evans C, Evans RM, Gerrard GE, et al. British Thyroid Association Guidelines for the Management of Thyroid Cancer. Clin Endocrinol. 2014;81:1-122.

32. Carty SE, Doherty GM, Inabnet WB, Pasieka JL, Randolph GW Shaha AR, et al. American Thyroid Association statement on the essential elements of interdisciplinary communication of perioperative information for patients undergoing thyroid cancer surgery. Thyroid. 2012;22:395-399.

33. Verkooijen RB, Verburg FA, Van-Isselt JW, Lips CJ, Smit JW, Stokkel MP The success rate of I-131 ablation in differentiated thyroid cancer: comparison of uptake-related and fixed-dose strategies. Eur $\mathrm{J}$ Endocrinol. 2008:159:301-307.

34. Robbins RJ, Schlumberger MJ. The evolving role of 131 for the treatment of differentiated thyroid carcinoma. J Nucl Med. 2005;46:28S-37S

35. Schvartz C, Bonnetain F, Debakuyo S, Gauthier M, Cueff A, Fieffé S, et al. Impact on overall survival of radiactive iodine low-risk differentiated thyroid cancer patients. J Clin Endocrinol Metab. 2012;97:1526-1535.

36. Mallick U, Harmer C, Yap B, Wadsley J, Clarke S, Moss L, et al. Ablation with low-dose radioiodine and thyrotropin alfa in thyroid cancer. $\mathrm{N}$ Eng J Med. 2012;366:1674-1685

37. Cheng W, Ma C, Fu H, Li J, Chen S, Wu S, et al. Low- or high-dose radioiodine remnant ablation for differentiated thyroid carcinoma: a meta-analysis. J Clin Endocrinol Metab. 2013:98:1353-1360.

38. Fallahi B, Beiki D, Takavar A, Fard-Esfahani A, Gilani KA, Saghari M, et al. Low versus high radioiodine dose in postoperative ablation of residual thyroid tissue in patients with differentiated thyroid carcinoma: a large randomized clinical trial. Nucl Med Commun. 2012;33:275-282.

39. Shah MD, Hall FT, Eski SJ, Witterick IJ, Walfish PG, Freeman JL. Clinical course of thyroid carcinoma after neck dissection. Laryngoscope. 2003:113:2102-2107.

40. Ruel E, Thomas S, Dinan M, Perkins JM, Roman SA, Sosa JA. Adjuvant radiactive iodine therapy is associated with improved survival for patients with intermediate-risk papillary thyroid cancer. J Clin Endocrinol Metab. 2015,100:1529-1536

41. Lamartina L, Durante C, Filetti S, Cooper DS. Low-risk differentiated thyroid cancer and radioiodine remnant ablation: a systematic review of the literature. J Clin Endocrinol Metab. 2015;100:1748-1761.

42. Sabra M, Grewal RK, Ghossein RM, Tuttle RM. Higher administered activities of radiactive iodine are associated with less structural persistent response in older, but not younger, papillary thyroid cancer patients with lateral neck lymph node metastases. Thyroid. 2014;24:1088-1095.

43. Schlumberger M, Tubiana M, De-Vathaire F, Hill C, Gardet P, Travagli JP et al. Long-term results of treatment of 283 patients with lung and bone metastases from differentiated thyroid carcinoma. J Clin Endocrinol Metab. 1986;63:960-967

44. Van-Nostrand D, Atkins F, Yeganeh F, Acio E, Bursaw R, Wartofsky L. Dosimetrically determined doses of radioiodine for the treatment of metastatic thyroid carcinoma. Thyroid. 2002;12:121-134.

45. Beckers C, Van-Ypersele-De-Strihou C, Coche E, Troch R, Malvaux P. lodine metabolism in severe renal insufficiency. J Clin Endocrinol Metab. 1969;29:293-296.

46. Tuttle M, Lebeauf R, Robbins RJ, Qualey R, Pentlow K, Larson SM, et al. Empiric radiactive iodine dosing regimens frequently exceed maximum tolerated activity levels in elderly patients with thyroid cancer. J Nucl Med. 2006; $47: 1587-1591$

47. Avram AC. Radioiodine scintigraphy with SPECT/CT: an important diagnostic tool for thyroid cancer staging and risk stratification. J Nucl Med. 2012;53:754-764.

48. Avram AM, Doherty GM, Fig LM, Wong K. Diagnostic 131-I fusion SPECT-CT Imaging in postoperative thyroid cancer patients: what is the impact on staging? Thyroid. 2011;21:193.

49. Brenner $\mathrm{W}$. Is thyroid stunning a real phenomenon or just fiction? J Nucl Med. 2002;43:835-836.
50. Guiraud-Vitaux F, Feldmann G, Vadrot N, Charles-Gupta S, Durand-Schneider AM, Colas-Linhart N, et al. Early ultrastructural injuries in the thyroid of the normal rat radioinduced by diagnostic and/or therapeutic amounts of iodine-131. Cell Mol Biol (Noisy-le-grand). 2001;47:495-502.

51. Kao CH, Yen TC. Stunning effects after a diagnostic dose of iodine-131. Nuklearmedizin. 1998;37:30-32

52. Aide N, Heutte N, Rame JP, Rousseau E, Loiseau C, Henry-Amar M, et al. Clinical relevance of single-photon emission computed tomography/ computed tomography of the neck and thorax in postablation 131I scintigraphy for thyroid cancer. J Clin Endocrinol Metab. 2009;94:2075-2084.

53. Ciappuccini R, Heutte N, Trzepla G, Rame JP, Vaur D, Aide N, et al. Postablation (131)I scintigraphy with neck and thorax SPECT-CT and stimulated serum thyroglobulin level predict the outcome of patients with differentiated thyroid cancer. Eur J Endocrinol. 2011;164:961-969.

54. Tuttle RM, Tala $H$, Shah J, Leboeuf R, Ghossein R, Gonen M, et al. Estimating risk of recurrence in differentiated thyroid cancer after total thyroidectomy and radiactive iodine remnant ablation: using response to therapy variables to modify the initial risk estimates predicted by the new American Thyroid Association staging system. Thyroid. 2010; 20:1341-1349

55. Vaisman F, Shaha A, Fish S, Tuttle RM. Initial therapy with either thyroid lobectomy or total thyroidectomy without radiactive iodine remnant ablation is associated with very low rates of structural disease recurrence in properly selected patients with differentiated thyroid cancer. Clin Endocrinol (Oxf). 2011;75:112-119.

56. Webb RC, Howard RS, Stojadinovic A, Gaitonde DY, Wallace MK, Ahmed J, et al. The utility of serum thyroglobulin measurement at the time of remnant ablation for predicting disease-free status in patients with differentiated thyroid cancer: a metaanalysis involving 3947 patients. $\mathrm{J}$ Clin Endocrinol Metab. 2012;97:2754-2763.

57. Latrofa F, Ricci D, Montanelli L, Rocchi R, Piaggi P, Sisti E, et al. Lymphocytic thyroiditis on histology correlates with serum thyroglobulin autoantibodies in patients with papillary thyroid carcinoma: impact on detection of serum thyroglobulin. J Clin Endocrinol Metab. 2012; 97:2380-2387.

58. Görges R, Maniecki M, Jentzen W, Sheu SN, Mann K, Bockisch A, et al. Development and clinical impact of thyroglobulin antibodies in patients with differentiated thyroid carcinoma during the first 3 years after thyroidectomy. Eur J Endocrinol. 2005;153:49-55.

59. Spencer CA. Clinical review: clinical utility of thyroglobulin antibody $(\mathrm{TgAb})$ measurements for patients with differentiated thyroid cancers (DTC). J Clin Endocrinol Metab. 2011;96:3615-3627.

60. Kim WG, Yoon JH, Kim WB, Kim TY, Kim EY, Kim JM, et al. Change of serum antithyroglobulin antibody levels is useful for prediction of clinical recurrence in thyroglobulin-negative patients with differentiated thyroid carcinoma. J Clin Endocrinol Metab. 2008:93:4683-4689.

61. Palaniswamy SS, Subramanyam P. Diagnostic utility of PETCT in thyroid malignancies: an update. Ann Nucl Med. 2013;27:681-693.

62. Feine U, Lietzenmayer R, Hanke JP, Held J, Wöhrle H, Müller-Schauenburg W. Fluorine-18-FDG and iodine-131-iodide uptake in thyroid cancer. J Nucl Med. 1996:37:1468-1472.

63. Schönberger J, Rüschoff J, Grimm D, Marienhagen J, Rümmele P, Meyringer R, et al. Glucose transporter 1 gene expression is related to thyroid neoplasms with an unfavorable prognosis: an immunohistochemical study. Thyroid. 2002;12:747-754.

64. Salvatori M, Biondi B, Rufini V. Imaging in endocrinology: 2-[18F]-fluoro2-deoxy-D-glucose positron emission tomography/computed tomography in differentiated thyroid carcinoma: clinical indications and controversies in diagnosis and follow-up. Eur J Endocrinol. 2016;173:115-130.

65. Pacak K, Eisenhofer G, Goldstein DS. Functional imaging of endocrine tumors: role of positron emission tomography. Endoc Rev. 2004;25:568-580.

66. Wang W, Larson SM, Fazzari M, Tickoo SK, Kolbert K, Sgouros G, et al. Prognostic value of [18F]fluorodeoxyglucose positron emission tomographic scanning in patients with thyroid cancer. J Clin Endocrinol Metab. 2000;85:1107-1113

67. Leboulleux S, Schroeder PR, Schlumberger M, Ladenson PW. The role of PET in follow-up of patients treated for differentiated epithelial thyroid cancers. Nat Clin Pract Endocrinol Metab. 2007:3:112-121.

68. Grabellus F, Worm K, Schmid KW, Sheu SY. The BRAFV600E mutation in papillary thyroid carcinoma is associated with glucose transporter 1 overexpression. Thyroid. 2012;22:377-382.

69. Xing M, Alzahrani AS, Carson KA, Viola D, Elisei R, Bendlova B, et al. Association between BRAF V600E mutation and mortality in patients with papillary thyroid cancer. JAMA. 2013;309:1493-1501.

70. Nagarajah J, Ho AL, Tuttle RM, Weber WA, Grewal RK, et al. Correlation of BRAFV600E mutation and glucose metabolism in thyroid cancer patients: an 18F-FDG PET study. J Nucl Med. 2015;56:662-666

71. Stokkel MP, Duchateau CS, Drogoiescu C. The value of FDG-PET in the follow-up of differentiated thyroid cancer: a review of the literature. $Q \mathrm{~J}$ Nucl Med Mol Imaging. 2006;50:78-87. 